http://portaldeperiodicos.unisul.br/index.php/EeN/index

\title{
UM ESTUDO SOBRE TÉCNICAS DE MAPEAMENTO DA COGNIÇÃO ORGANIZACIONAL: REPRESENTAÇÕES SOCIAIS VERSUS SELF Q
}

\section{A STUDY ON ORGANIZATIONAL COGNITION MAPPING TECHNIQUES: SOCIAL REPRESENTATIONS VERSUS SELF Q \\ UN ESTUDIO SOBRE TÉCNICAS DE COGNICIÓN ORGANIACIONAL: REPRESENTACIONES SOCIALES VERSUS SELF Q}

\section{Lasier Gorziza de Souza}

Doutorando em Administração pela Unisinos

Endereço: Rua Do Guia Lopes, 867/82, Centro, CEP: 96020-392. Caxias do Sul, RS, Brasil

Telefone: (054) 3533-6299

E-mail: lasier@uol.com.br

\section{Heitor José Cademartori Mendina}

Doutorando em Administração pela Unisinos

Endereço: Rua Ari Baierli, 85/404, CEP: 95760-000. São Sebastião do Caí, RS, Brasil

Telefone: (051) 3635-4070

E-mail: heitormendina@hotmail.com

\section{Gabriel Sperandio Milan}

Professor e Pesquisador da Universidade de Caxias do Sul (UCS)

Doutor em Engenharia de Produção pela Universidade Federal do Rio Grande do Sul (UFRGS)

Endereço: Rua Francisco Getúlio Vargas, 1130, Bloco F / PPGA.CEP: 95070-560. Caxias do Sul, RS, Brasil Telefone: (054) 3218.2100

E-mail: gsmilan@ucs.br

\section{Claudia Cristina Bitencourt}

Professora e Pesquisadora da Universidade do Vale do Rio dos Sinos (Unisinos) Doutora em Administração pela Universidade Federal do Rio Grande do Sul (UFRGS)

Endereço: Av. Unisinos, 950, Centro de Ciências Econômicas - PPG Administração, CEP: 93022-000. São

Leopoldo, RS, Brasil

Telefone: (051) 8111-2273 ou (051) 3590-8186

E-mail: claudiacb@unisinos.br

Artigo recebido em 01/11/2013. Revisado por pares em 25/02/2014. Reformulado em 21/06/2014. Recomendado para publicação em 21/06/2014 por Ademar Dutra (Editor Científico). Publicado em 29/08/2014. Avaliado pelo Sistema double blind review.
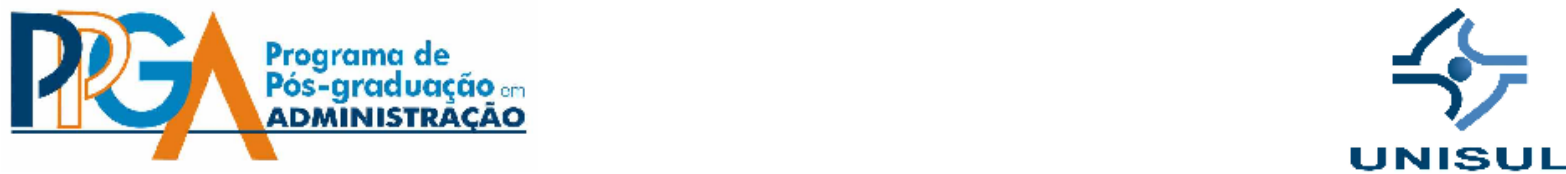

\section{(i) $(9)$}




\section{RESUMO}

Este trabalho se propõe a analisar, a partir do estudo desenvolvido por Nicolini (1999), duas metodologias de pesquisa que abordam a cognição organizacional. Utilizando-se da Teoria das Representações Sociais de Serge Moscovici, e dos Mapas Causais (Self Q) de Michael Bougon, aplicou-se uma pesquisa em uma indústria exportadora de alimentos, um frigorífico que se dedica ao abate de aves (frangos), possibilitando a coleta dos dados para posterior análise por meio de ambas as metodologias. Assim, dois mapas foram construídos, um utilizando a técnica das Representações Sociais e o outro com aplicação da técnica Self Q. Os resultados demonstraram que características captadas pelas duas metodologias foram semelhantes e convergentes, resguardando-se as peculiaridades de cada método.

Palavras-chave: Teoria das Representações Sociais; Self Q; Mapas causais; Psicologia cognitiva; Cognição organizacional.

\section{ABSTRACT}

This work aims to analyze, from the study developed by Nicolini (1999), two research methodologies addressed to the organizational cognition. Using the Social Representations Theory developed by Serge Moscovici, and Causal Mapping (Self Q), developed by Michael Bougon, it was applied a research in a food export industry, a meatpacking industry dedicated to slaughter of chickens, making possible the data collection for later analyze through both methods. Thus, two maps were constructed, one of them using a technique of social representations and other one with Self $Q$ technical application. The results showed that characteristics captured by the two methods were similar and convergent, preserving the peculiarities of each method.

Keywords: Social Representations Theory; Self Q; Causal mapping; Cognitive Psychology; Organizational cognition.

\section{RESUMEN}

Este trabajo se propone a analizar, desde el estudio desarrollado por Nicolini (1999), dos metodologías de investigación que abordan la cognición organizacional. Utilizándose de la Teoría de las Representaciones Sociales de Serge Moscovici y de los Mapas Causales (Self Q), de Michael Bougon se aplicó una pesquisa en una industria exportadora de alimentos, un frigorífico que se dedica al sacrificio de aves (pollos), posibilitando la colección de los datos para posterior análisis y por medio de ambas metodologías. Así, dos mapas fueron construidos, un utilizándosele la técnica de las Representaciones Sociales y otro con aplicación de la técnica Self $Q$. Los resultados demostraran que características captadas por las dos metodologías fueron similares y convergentes, resguardándosele las peculiaridades de cada método.

Palabras-clave: Teoría de las Representaciones Sociales; Self Q; Mapas causales; Psicología cognitiva; Cognición organizacional. 


\section{INTRODUÇÃO}

Os estudos de Barnard (1938) enfatizaram que o indivíduo costuma agir dentro das organizações condicionado pela maneira de como ele percebe o mundo exterior, fato que possibilita um entendimento subjetivo do ambiente organizacional. Embora este conceito tenha estabelecido uma linha de abordagem em relação à cognição organizacional, é na década de 80 e início dos anos 90 que este campo de estudo desperta maior interesse dos pesquisadores. No entender de Nicolini (1999), tal interesse nos processos cognitivos dentro das organizações deve-se, também, à introdução da noção de ator organizacional inserida nos estudos de March e Simon (1958) e Cyert e March (1963).

Por outro lado, o recente aumento do interesse na cognição organizacional temse desenvolvido apoiando-se nos esteios da psicologia cognitiva e do comportamento organizacional. Schneider e Angelmar (1993) destacam que, por trás deste crescimento, está um silogismo simplista, ou seja, as pessoas pensam, e como os gerentes são pessoas, logo os gestores também pensam. Uma vez que estes processos cognitivos ocorrem em um ambiente organizacional, estes estudos podem ser referidos como a análise dos processos cognitivos nas organizações.

A noção de que é possível compreender fenômenos organizacionais, usando a mente e o cérebro humano como metáforas (MORGAN, 1986), estimulou o desenvolvimento de metodologias para a análise da cognição organizacional. Segundo Nicolini (1999), este esforço para compreender e descrever tais fenômenos organizacionais, em termos cognitivos, levou à criação de vários métodos e técnicas com os quais é possível investigar os processos cognitivos de membros (indivíduos) e organizações. Pesquisas e análises destes métodos têm sido propostas por Huff (1990), Fiol e Huff (1992), Nicolini, (1995), Walsh (1995), Strati e Nicolini (1997) e Eden e Spender (1998), dentre outros.

Devido à singularidade do objeto sob observação, a construção de métodos para capturar e representar a cognição organizacional sempre foi especialmente difícil. Muitas vezes, os problemas estão centrados em torno da validade do método, ou seja, nas questões metodológicas, e também na eficácia das técnicas propostas. Desta forma, as perguntas 
direcionam-se sobre o quê e como estes métodos de análise são capazes de capturar os processos de percepção de sentido dos membros (partícipes) de uma organização.

Na medida em que a validade do método está em questão, uma série de estudiosos argumenta que o uso de uma metodologia adequada torna possível capturar e representar as construções mentais, que efetivamente guiam os atores organizacionais em seus comportamentos no cotidiano (HUFF, 1990; FIOL; HUFF, 1992; WALSH, 1995). Na visão dos pesquisadores, indivíduos e organizações processam informações do ambiente usando alguma forma estruturada do conhecimento, ou melhor, um modelo mental em que os indivíduos impõem sobre uma informação dando forma e, logicamente, significado (WALSH, 1995). As representações simbólicas e estruturas mentais, portanto, têm papel central no contexto de toda esta abordagem.

Ao mesmo tempo, Nicolini (1999) entende que as representações simbólicas e estruturas mentais desempenham um papel de mediação entre estímulo e resposta, de modo que o objetivo da abordagem é atribuir propriedades causais ao desejo das pessoas, às suas convicções e à sua vontade, isto é, estabelecer se elas são representativas ou intencionais, acima de tudo, fisicamente possíveis e capazes de gerar comportamentos.

No entanto, há um pequeno número de estudiosos que têm levantado dúvidas sobre a possibilidade de mapeamento cognitivo das estruturas organizacionais. Narayan e Fahey (1990), por exemplo, sugerem que a correlação entre a percepção do verdadeiro não é perfeita em mapas causais, pois é moldada pela natureza do discurso público e do contexto no qual ele ocorre. Em outras palavras, Nicolini (1999) acredita que a natureza do contexto da divulgação e da presença de um bom motivo para esconder o conteúdo do mapa, como, por exemplo, em uma situação altamente competitiva, pode interferir com o esforço do pesquisador para determinar a natureza das lentes que filtram dados e constituem o meio pelo qual estes são analisados e interpretados.

Finalmente, ainda há um grupo de autores que adotam uma postura mais cautelosa, ontológica e epistêmica para toda a questão do mapeamento da cognição organizacional. Axelrod (1976), um dos pioneiros na construção de mapas causais, aparentemente não desconsidera a possível existência de estruturas de conhecimento do 
grupo, excluindo, a priori, a possibilidade de capturar os processos de pensamento dos atores organizacionais por meio de ferramentas analíticas. Segundo o autor, o termo cognitivo na expressão mapas cognitivos não deve nos enganar; uma vez que os mapas não são uma forma capaz de se produzir inferências sobre o que os tomadores de decisão organizacional, ou atores, realmente acreditam, eles apenas dão ordem e representam graficamente as suas declarações.

Nessa linha de pensamento, Nicolini (1999) entende que os mapas devam ser considerados apenas como instrumentos de descrição e de representação que ajudam os modos de discussão e análise de algumas correntes de pensamento na explicação dos acontecimentos. No entanto, o autor destaca que os mapas são extremamente úteis, porque descrevem as formas em que certas classes de fenômenos são interpretadas, tornando-se base para uma experiência de autorreflexão que produz o aprendizado e a mudança.

O objetivo do trabalho de Nicolini (1999), portanto, não foi o de validar ou refutar a existência de mapas ou representações ou, ainda, o de explorar até que ponto alguns dos métodos de mapeamento de cognição organizacional podem ajudar a aprofundar nossa compreensão da dimensão cognitiva do processo organizacional. Neste sentido, o autor se propôs a investigar o que os diferentes métodos permitem ver, em relação à sua capacidade de fornecer descrições relevantes dos aspectos cognitivos dos processos das organizações, e o equilíbrio entre o que tais metodologias destacam ou ocultam, bem como identificar os compromissos, as ambiguidades e as contradições que estes métodos têm para se sustentar, a fim de produzir suas respectivas representações da realidade.

Este trabalho, portanto, propõe-se a replicar o estudo desenvolvido por Nicolini (1999), visto que este autor, nas limitações de seu trabalho, fez uma alusão à necessidade de mais comparações e, em outros contextos, entre dois métodos. Para tanto, os autores do presente estudo propuseram a comparação entre duas metodologias, abordando os mapas cognitivos por meio da utilização das técnicas das Representações Sociais e do Self Q. Desta forma, o estudo apresenta uma comparação das duas metodologias de pesquisa, as quais são descritas detalhadamente a seguir.

Revista Eletrônica de Estratégia \& Negócios, Florianópolis, v.7, n.2, mai./ago. 2014. 


\section{REFERENCIAL TEÓRICO E METODOLOGIAS DE PESQUISA EMPREGADAS}

Como dito anteriormente, este estudo foi elaborado a partir do trabalho de Nicolini (1999), no qual se propôs testar duas diferentes metodologias de análise cognitiva das organizações, com a intenção de observar, entender e destacar as consequências decorrentes da diversidade de cada abordagem e, possivelmente, dos diferentes entendimentos sobre os aspectos cognitivos dos processos organizacionais.

Neste contexto, o presente trabalho, assim como o de Nicolini (1999), comparou duas metodologias de pesquisa da cognição organizacional, ou seja, contrapôs a técnica francesa das Representações Sociais através da Teoria das Representações Sociais (TRS) (MOSCOVICl, 1969) versus os mapas causais, empregando a técnica de autoquestionamento, o Self Q, criada por Bougon, Weick e Binkhorst (1977).

A Teoria das Representações Sociais (TRS), introduzida pelo psicólogo social Serge Moscovici, compreende a análise quantitativa de dados textuais coletados por entrevistas semiabertas (ou semiestruturadas) (RIBEIRO; MILAN, 2004; KING; HORROCKS, 2010), e decorre da tradição da psicologia e da sociologia social francesa de Durkheim (MOSCOVICI, 1969; 1981; 1984; 1985; 1987; 1988; 2000; FARR; MOSCOVICI, 1984; ABRIC, 1984; 1993; 1994; 1996; DI GIACOMO, 1985; FARR, 1987; 1993; JODELET, 1989; 1991; 2001).

Os mapas causais construídos de acordo com as indicações teóricas de Bougon (1983; 1986; 1992) e Bougon et al. (1990) são baseados em ciclos de autoquestionamentos, que combinam, de forma não tradicional, elementos da psicologia cognitiva com conceitos derivados da fenomenologia, da cibernética e da Teoria do Sistema (BOUGON, 1992).

Enquanto um método está focado na captura de padrões inter-relacionados da percepção individual, como é o caso das Representações Sociais, o outro se esforça para obter conteúdo, forma e função dos processos coletivos idealizados por meio da comunicação e do diálogo entre indivíduos (MOSCOVICl, 1985).

Por fim, solicitou-se aos participantes que expressassem suas próprias opiniões sobre as diversidades dos trabalhos produzidos. Portanto, este estudo é um exemplo de 
pesquisa qualitativo-exploratória (HENNINK; HUTTER; BAILEY, 2011; SCOTT; GARNER, 2013) para comparar duas metodologias diferentes em ação, dentro da análise cognitiva de um determinado campo da organização.

\section{AMBIÊNCIA DE PESQUISA}

A pesquisa foi realizada em uma indústria exportadora de alimentos com mais de 30 anos de atuação no mercado, localizada no Vale dos Sinos, no Estado do Rio Grande do Sul (RS). Trata-se de um frigorífico que se dedica ao abate de aves, sendo que, no período da aplicação deste estudo, apresentava uma capacidade operacional (de abate) de 65 mil frangos/dia. Em relação à sua força de trabalho, a unidade industrial, denominada aqui por "Q-Frango", no sentido de preservar identidade da empresa, empregava 860 colaboradores.

A pesquisa deste estudo contou com 21 participantes, que foram divididos em três grupos homogêneos na composição de suas características. Para tanto, era necessário ter pelo menos três anos de trabalho na empresa, paridade de papéis quanto às atividades profissionais desempenhadas e, no mínimo, Ensino Médio completo. Ressalta-se que todos os envolvidos atuavam em cargos de média gerência e participaram de maneira voluntária.

$\mathrm{Na}$ apresentação das atividades a cada grupo de trabalho, foram explicados verbalmente os objetivos da pesquisa aos entrevistados, por meio do enunciado vamos comparar duas técnicas destinadas a representar certos aspectos da empresa. Também foi enfatizado o âmbito puramente acadêmico que o estudo se propunha, bem como o compromisso com o sigilo em relação à autoria das percepções e opiniões de cada um.

No entanto, apenas dois dos grupos construíram as representações gráficas das respectivas metodologias apresentadas a seguir, trabalhando separadamente, e cada um utilizando-se de um único método. O terceiro grupo teve como finalidade servir de apoio e discussão, e só participou da última reunião, a de avaliação geral dos resultados. Nesta oportunidade foram apresentados e discutidos os dois mapas com todos os participantes.

Os dois grupos receberam os mesmos estímulos para trabalharem na construção dos mapas da cognição organizacional e da representação social. Na tentativa de obter as 
categorias fundamentais usadas pelos membros da organização, e para dar sentido à percepção dos processos organizacionais de liderança, os participantes foram convidados a responder o seguinte questionamento: Quais características são necessárias para um líder trabalhar na empresa Q-Frango? Isto é, para ser aprovado, aceito e bem-sucedido em seu trabalho? Em seguida, os participantes responderam ao questionamento de acordo com o procedimento estabelecido pelas respectivas metodologias.

Após o final de cada etapa de trabalho, a representação obtida nas respectivas metodologias foi mostrada aos membros dos grupos que as elaboraram. Na fase seguinte, os participantes, de maneira individual, foram convidados a responder um sucinto questionário sobre o grau de satisfação percebido em relação ao mapa produzido por sua equipe de trabalho. Posteriormente, todos os três grupos foram convidados para uma sessão plenária de apresentação dos mapas obtidos, a partir de cada uma das duas metodologias empregadas. Neste momento, eles foram convidados e estimulados a expressarem suas opiniões sobre as diferenças percebidas entre as representações resultantes.

\section{DESENVOLVIMENTO DA PESQUISA}

\subsection{A CONSTRUÇÃO DO MAPA DE REPRESENTAÇÕES SOCIAIS (MÉTODO 1)}

Utilizando-se os pesquisadores do papel de entrevistadores, o primeiro método foi aplicado permitindo a construção de representações sociais sobre a base de dados textuais, recolhidas a partir de entrevistas semiabertas (ou semiestruturadas), realizadas individualmente com cada participante do estudo. A premissa básica deste método é a noção de representação social, introduzida nos anos 60 pelo psicólogo social francês Serge Moscovici. Este autor defendeu a existência de universos socialmente determinados de opinião, isto é, as representações sociais, que funcionam tanto em nível cognitivo, na medida em que propõem expressões consensualmente validadas e simbólicas das relações sociais, quanto em nível comportamental, quando ajudam a orientar a ação coletiva direta (MOSCOVICl, 1969; 1984; 1988; 2000). De acordo com o autor, as representações sociais são um sistema cognitivo em nível social, que permite que a realidade venha a ser organizada e aproveitada. Por meio de comunicação, os indivíduos e os grupos conferem uma realidade 
física de ideias e imagens, por meio dos sistemas de classificação e denominação das suas representações.

Em suma, as representações sociais podem ser definidas como a elaboração de um objeto por uma comunidade, que permite aos seus membros se comportarem e se comunicarem de forma compreensível. Eles são sistemas cognitivos da linguagem e com uma lógica que permite, aos membros de uma determinada comunidade, estabelecer as condições e os contextos de suas interações. As representações sociais permitem aos indivíduos e grupos construírem uma visão coerente da realidade, que eles usam para orientar seu comportamento. Portanto, elas são o resultado da atividade mental modulada pelas características da situação social em que são produzidas (GHERARDI, 1998).

Segundo alguns autores, as representações sociais articulam-se em torno de um núcleo central, rodeado por elementos periféricos (ABRIC, 1984; 1993; 1994; DI GIACOMO, 1985; FARR, 1993). Este núcleo consiste no componente de atitude, e pode ser chamado de local de coerência. É o principal organizador da representação, o elemento discriminador em relação aos quais todos os elementos da representação são organizados e dotados de significados. Dadas estas características, o núcleo central é a parte mais estável de uma representação social, isto é, aquele que mais se opõe às mudanças (ABRIC, 1984). Sendo assim, as representações sociais podem ser entendidas como um conjunto de conceitos, proposições e explicações originados na vida cotidiana no curso das comunicações interpessoais, e equivalem aos mitos e aos sistemas de crenças na sociedade, podendo ser vistas, também, como a versão contemporânea do senso comum. Referindo-se à organização interna das representações sociais, identifica-se a Teoria do Núcleo Central, a qual postula que o homem organiza e processa as informações de forma dinâmica e evolutiva em torno de um núcleo central e de um conjunto de elementos periféricos (ABRIC, 1984; 1993; 1994; 1996; SÁ, 1996; 1998; MOSCOVICI, 1981; 1984; 1987; 1988; 2000).

Nicolini (1999) afirma que a rede de significados atribuídos a uma representação dá origem a um campo de representação. Este, por sua vez, é um mapa fidedigno que restaura o objeto da representação reconstruída de acordo com a natureza, necessidades e crenças de determinado grupo de indivíduos, cujo interesse tenha despertado. Desta forma, 
configuram evidências específicas do campo da coerência e do campo da representação a partir da avaliação e da seleção das informações coletadas sobre o objeto representado.

Segundo os autores, que primeiro introduziram o termo Representação Social, a melhor maneira de se identificar tais reproduções é com a realização de algum tipo de análise de conteúdo de textos escritos ou orais (FARR; MOSCOVICl, 1984). Na verdade, este é o método geralmente empregado para identificar as representações sociais na tradição francesa. Neste estudo, porém, foi utilizada uma técnica quantitativa de análise de texto, conhecida como análise de similaridade ou análise de similitude, baseando-se na computação de coocorrência de dados textuais (DEGENNE; VERGÈS, 1973), dados estes provenientes das evocações dos entrevistados (VERGÈS, 1992; VERGÈS; TYSZKA, 1994).

No entendimento de Nicolini (1999), a análise de similaridade é uma técnica quantitativa pela qual é possível destacar as relações significativas entre as partes de um todo. Introduzida para estudar as representações sociais, a técnica é baseada no pressuposto fundamental de que dois temas são cognatos se os sujeitos os utilizam juntos. 0 método, portanto, mede a coocorrência entre os elementos do texto, e representa os resultados em um gráfico plotado a partir das relações que ligam pares de variáveis. Embora a análise de similaridade seja capaz de capturar apenas uma dimensão das relações complexas entre as representações sociais, ela tem sido frequentemente utilizada para este fim, pois oferece a vantagem de não exigir qualquer hipótese preliminar sobre a natureza das relações entre os elementos (FLAMENT, 1981; 1987; 1994a; 1994b).

Neste estudo, os dados foram coletados mediante entrevistas realizadas com os participantes. Os pesquisadores propuseram a questão inicial, usada para todos os membros dos dois primeiros grupos. Em seguida, o processo foi apoiado por perguntas abertas para complementar cada etapa da pesquisa. As entrevistas foram gravadas, transcritas e codificadas pelos pesquisadores (RIBEIRO; MILAN, 2004) com base em uma série de dimensões de interesse em relação ao tema em análise. Na etapa seguinte, os códigos similares utilizados pelos entrevistados, ao se referirem a uma mesma dimensão, foram abstraídos, sintetizados e categorizados, configurando-se como análise de conteúdo (BARDIN, 2004; SCHREIER, 2012). 
Desta forma, foi possível avançar para a etapa de elaboração quantitativa, bem como calcular a frequência absoluta de categorias, revelando, assim, o número de vezes que os indicadores empíricos apareceram. O exame da copresença de indicadores nas entrevistas individuais proporcionou os índices de coocorrência, permitindo que as relações representativas entre as variáveis fossem descritas. O resultado foi uma árvore hierárquica de relações, ou seja, a árvore máxima de diferentes índices de coocorrência entre as categorias identificadas.

Para tanto, a Figura 1 exibe a representação gráfica da árvore máxima, a qual retrata tanto o nível de coocorrência das categorias, quanto o das frequências individuais identificadas nas entrevistas. As frequências das categorias são representadas pela amplitude ao se diminuir o tamanho dos círculos. A força do índice de coocorrência é concebida pelo número de linhas que conectam as categorias, quando estas ocorrem. Ao se combinar os dois critérios, organizam-se as categorias mais frequentes em uma conexão mais estreita no centro, o núcleo da representação social torna-se imediatamente aparente.

Assim sendo, pela Teoria das Representações Sociais (TRS), identificou-se que as principais características exigidas para um líder trabalhar na empresa Q-Frango, isto é, para ser aprovado, aceito e bem-sucedido no seu ambiente de trabalho está, principalmente, organizada em torno de um núcleo sólido de categorias de acoplamento em relação ao compromisso com a execução das tarefas operacionais, bem como com os princípios das relações humanas no trabalho.

Analisando a Figura 1, percebe-se que as categorias mais frequentes e com número maior de coocorrência referem-se às seguintes categorias: Ser responsável com as tarefas da empresa (O), Ouvir com atenção (E) e Ser bem-educado (afável) com as pessoas (L). Destacam-se, ainda: Ter paciência com os outros e com os problemas (R) e Inspirar confiança (C) como as categorias correlacionadas e que coocorreram com o núcleo da representação social, porém, identificadas como integrantes da periferia. Um pouco mais afastadas do núcleo, contudo, correlacionadas com a categoria pertencente ao núcleo central, emergiu Ouvir com atenção (E), e apareceram três outras categorias. São elas: Ser honesto com as pessoas (N), Proporcionar o diálogo (G) e Delegar tarefas (B). Por último, 
destacam-se duas categorias correlacionadas entre si: Agilidade nas decisões (A) e Ter a visão do todo $(\mathrm{P})$, que obtiveram duas coocorrências, todavia, não correlacionadas com núcleo central da representação social.

Entre as categorias identificadas como participantes do núcleo central, portanto, onde haverá maior resistência aos líderes integrantes no contexto deste estudo que não apresentarem tais características, destacam-se a intercorrelação entre todas as categorias participantes, mas sobressaindo a correlação entre Ser bem-educado (afável) com as pessoas (L) com a categoria Ser responsável com as tarefas da empresa (0), que obtiveram quatro coocorrências. Por último, a categoria Ouvir com atenção (E) foi a que obteve o maior número de conexões correlacionadas, além de se correlacionar com as outras categorias do núcleo, obtendo mais cinco coocorrências, totalizando sete conexões.

Figura 1 - Representação social das competências de liderança

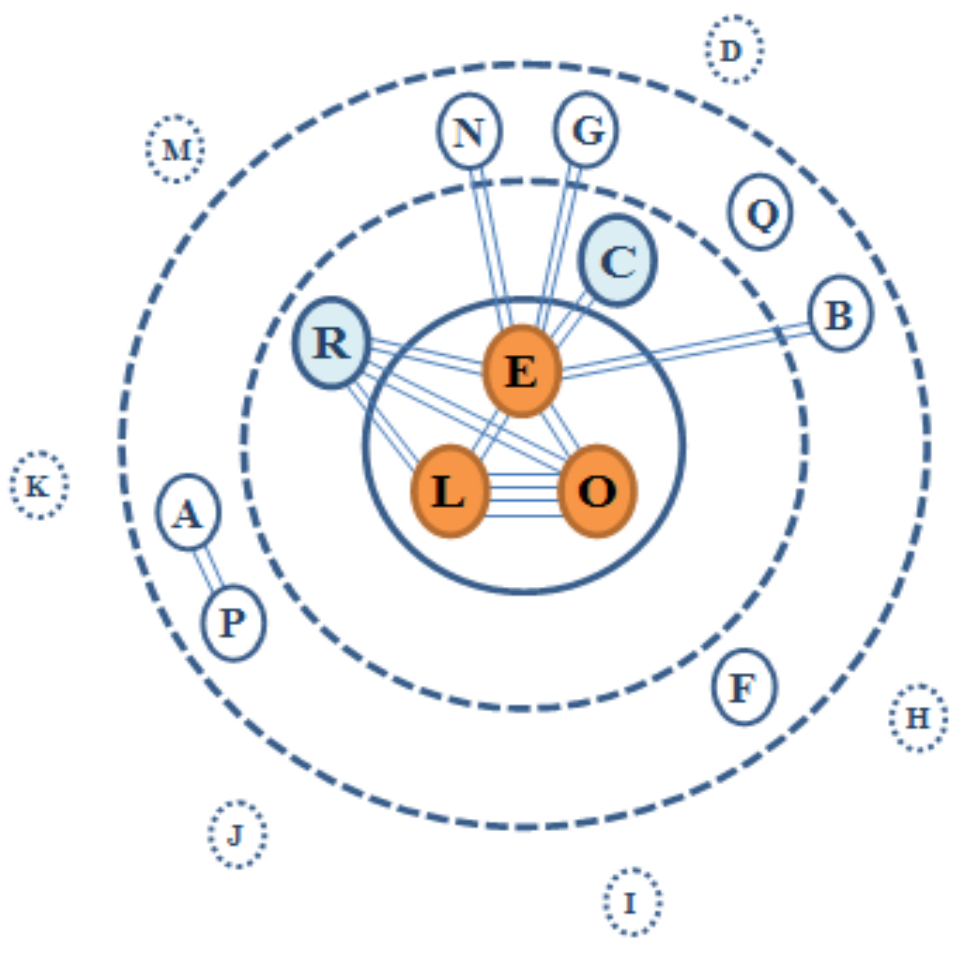

Revista Eletrônica de Estratégia \& Negócios, Florianópolis, v.7, n.2, mai./ago. 2014. 
UM ESTUDO SOBRE TÉCNICAS DE MAPEAMENTO DA COGNIÇÃO ORGANIZACIONAL Lasier Gorziza de Souza - Heitor José Cademartori Mendina - Gabriel Sperandio Milan - Claudia Cristina Bitencourt

\begin{tabular}{|c|c|}
\hline Legenda & Competências de Liderança Identificadas Junto aos Participantes \\
\hline A & Agilidade nas decisões \\
\hline B & Delegar tarefas \\
\hline $\mathbf{C}$ & Inspirar confiança \\
\hline D & Liderar pelo exemplo \\
\hline $\mathbf{E}$ & Ouvir com atenção \\
\hline $\mathbf{F}$ & Proporcionar bem-estar \\
\hline $\mathbf{G}$ & Proporcionar o diálogo \\
\hline $\mathbf{H}$ & Raciocinar antes de tomaruma decisão \\
\hline I & Respeitar os superiores \\
\hline $\mathbf{J}$ & Saber fazer (know how) \\
\hline $\mathbf{K}$ & Saber treinar e desenvolver pessoas \\
\hline $\mathrm{L}$ & Ser bem-educado (afável) com as pessoas \\
\hline $\mathbf{M}$ & Ser flexível nastomadas de decisões \\
\hline $\mathbf{N}$ & Ser honesto com as pessoas \\
\hline $\mathbf{O}$ & Ser responsável com as tarefas da empresa \\
\hline $\mathbf{P}$ & Ter a visão do todo \\
\hline Q & Ter interesse pelos problemas da empresa \\
\hline $\mathbf{R}$ & Ter paciência com os outros e com os problemas \\
\hline
\end{tabular}

\begin{tabular}{|cccc|}
\hline Tópicos & Frequência & Links & Coocorrência \\
\hline & 4 & $\overline{\bar{E}}$ & 4 \\
& 3 & $\overline{ }$ & \\
\hline & 2 & & \\
\hline
\end{tabular}

Fonte: Elaborada pelos autores a partir dos resultados da pesquisa.

\subsection{A CONSTRUÇÃO DO MAPA COGNITIVO ORGANIZACIONAL UTILIZANDO O SELF Q (MÉTODO 2)}

De acordo com Nicolini (1999), o Self Q é uma técnica que foi desenvolvida por Michael Bougon e seus colaboradores (BOUGON; WEICK; BINKHORST, 1977, BOUGON, 1983; 1986; 1992; BOUGON et al., 1990) para desenhar mapas causais, tanto individuais quanto organizacionais, sendo baseada na combinação de auto entrevistas com uma série de atividades estruturadas, a fim de identificar uma rede de conceitos conectados por relações

Revista Eletrônica de Estratégia \& Negócios, Florianópolis, v.7, n.2, mai./ago. 2014. 
causais sem exigir que o entrevistado gere uma representação completa. Segundo os autores, um mapa causal é uma forma particular de mapa cognitivo, compreendendo um conjunto de conceitos interligados por relações causais. Desta forma, tais mapas consistem em redes de conceitos conectados por operadores de lógica causal (BOUGON, 1983; 1986; 1992; WEICK; BOUGON, 1986; BOUGON et al., 1990).

Bougon (1983) destaca que a metodologia Self Q é não diretiva e não reativa, porque transfere grande parte da responsabilidade da organização, execução da coleta de dados e, por fim, a construção do mapa, do entrevistador para o entrevistado. Esta abordagem, entretanto, colabora na coleta de informações significativas. O método baseiase também na criação de um cenário próprio, que ajuda a evitar a tendência dos atores a repetirem individualmente as teorias proclamadas em níveis oficiais de suas organizações. Devido a certas características, como a minimização de interferências, o alto nível de feedback e o uso das próprias linguagens e expressões dos atores organizacionais, o Self Q é uma técnica cognitiva que se assemelha aos sofisticados métodos etnográficos de coleta de dados.

Cabe salientar que a construção de mapas cognitivos foi uma área específica de interesse para o estudo de Nicolini (1999), devido ao fato que aquele trabalho foi um dos primeiros, e poucos casos de aplicação literal da metodologia introduzida por Bougon, Weick e Binkhorst (1977), e posteriormente desenvolvida, tanto teórica quanto metodologicamente por Bougon et al (1990).

Durante a primeira entrevista, que foi conduzida em contexto individual e de forma não diretiva, foram reunidos os conceitos e noções de cada participante. As entrevistas procederam de forma anômala devido à metodologia utilizada, e os pesquisadores solicitaram aos entrevistados que formulassem suas próprias questões sobre o tema deste estudo, daí o nome da metodologia. Uma vez que os pontos de vista (percepções, opiniões) foram transformados pelos pesquisadores em conceitos e noções, preservando-se, no entanto, a redação original, os entrevistados foram solicitados a estabelecer as relações causais entre os conceitos, utilizando de um sistema de cruzamento simples. Na prática, os participantes foram solicitados a indicar quais noções influenciavam 
em outras, seja positiva ou negativamente, e em que medida, se pouco, consideravelmente ou muito. Com base nestes dados, os pesquisadores elaboraram os mapas individuais de cada um dos participantes.

Para a elaboração do mapa coletivo, a partir dos mapas individuais, matrizes de adjacência foram construídas e, em seguida, as matrizes de alcançabilidade entre conceitos foram identificados. Após esta etapa, os conceitos foram, então, ordenados de acordo com seu grau em relação às conexões de chegada e de saída, ou seja, as influências nas outras conexões no mapa. Para Nicolini (1999), a teoria proposta por Bougon, Weick e Binkhorst (1977), em relação aos conceitos de dependências com níveis elevados de conexões de entrada, deve ser interpretada como reportando o objetivo da ação descrita por outros atrelamentos. Desra forma, isso tornou possível incluir as conexões de acordo com um esquema em forma de rede, em que os fatores causais ou pressuposições ficaram mais à esquerda, os meios para o centro, e os efeitos ou metas à direita.

Bougon et al. (1990) afirmam que os mapas cognitivos individuais constituem uma única estrutura subjacente cognitiva, que é responsável pela organização e estratégias de ação de qualquer sistema social. O mapa compartilhado, no entanto, é altamente dinâmico e está constantemente em transformação, propagando-se e sendo constantemente renegociado. Além disso, é baseado em um pequeno número de conexões entre os mapas individuais. O acordo não é produzido em um nível profundo entre os mapas cognitivos dos membros da organização, apenas elementos superficiais conhecidos como expressões-rotuladas são envolvidos. Estes conceitos enigmáticos, ou seja, as expressõesrotuladas fornecem o material para as atividades de preparo e, no nosso caso, para os blocos de construção com os quais mapas coletivos puderam ser construídos a partir das percepções individuais.

No entendimento de Nicolini (1999), as conexões enigmáticas em um mapa correspondem às afirmações que, internamente no sistema social, interligam os processos organizacionais, possibilitando membros com objetivos diferentes coordenarem suas ações, a fim de alcançarem resultados mutuamente vantajosos. Os membros da organização devem, portanto, reconhecer que suas expressões são semelhantes, relacionáveis e, por 
vezes, substituíveis com as dos outros colegas, embora essa semelhança possa não resistir a mais profunda análise interpretativa. Assim sendo, é possível identificar as conexões enigmáticas pedindo aos atores para pesquisarem entre as conexões dos mapas dos outros participantes as expressões-rotuladas que eles consideram ser sinônimo de seus próprios conceitos. As declarações mais frequentes são aquelas selecionadas e reconhecidas como refletindo as expressões de muitos dos outros participantes. Logo, a partir da percepção de um determinado sujeito, elas podem ser consideradas como os pontos que se sobrepõem ao seu próprio mapa.

A fim de se identificar tais conexões enigmáticas, procedeu-se por um pedido individual aos próprios participantes, para indicarem qual das declarações feitas por outros participantes são substituíveis, ou são sinônimos, com os seus próprios conceitos. Para Nicolini (1999), no espírito do trabalho de Bougon, as conexões enigmáticas no mapa coletivo foram identificadas por meio do mesmo procedimento utilizado para construir os mapas individuais causais, ou seja, deliberadamente, evitando a interação aberta entre os participantes. Com base na aplicação do critério segundo as conexões que recebem o maior número de sinônimos, podem ser consideradas aquelas que melhor resumem o significado de todas as outras e que são, por conseguinte, mais suscetíveis de constituir as conexões enigmáticas.

As conexões mais enigmáticas foram aquelas com o mais elevado grau de sinonímia, porque melhor resumiram as características sob investigação. Identificado isso, os dados foram utilizados para construir o mapa cognitivo coletivo. Para tanto, as conexões mais enigmáticas foram colocadas nas mesmas posições que foram atribuídas pelos entrevistados em seus mapas individuais, e as ligações entre elas foram transpostas a partir desses mapas. O mapa cognitivo coletivo criado a partir das conexões enigmáticas identificadas por meio da metodologia acima é apresentado na Figura 2.

Revista Eletrônica de Estratégia \& Negócios, Florianópolis, v.7, n.2, mai./ago. 2014. 
Figura 2 - Mapa causal das competências de liderança - Self Q

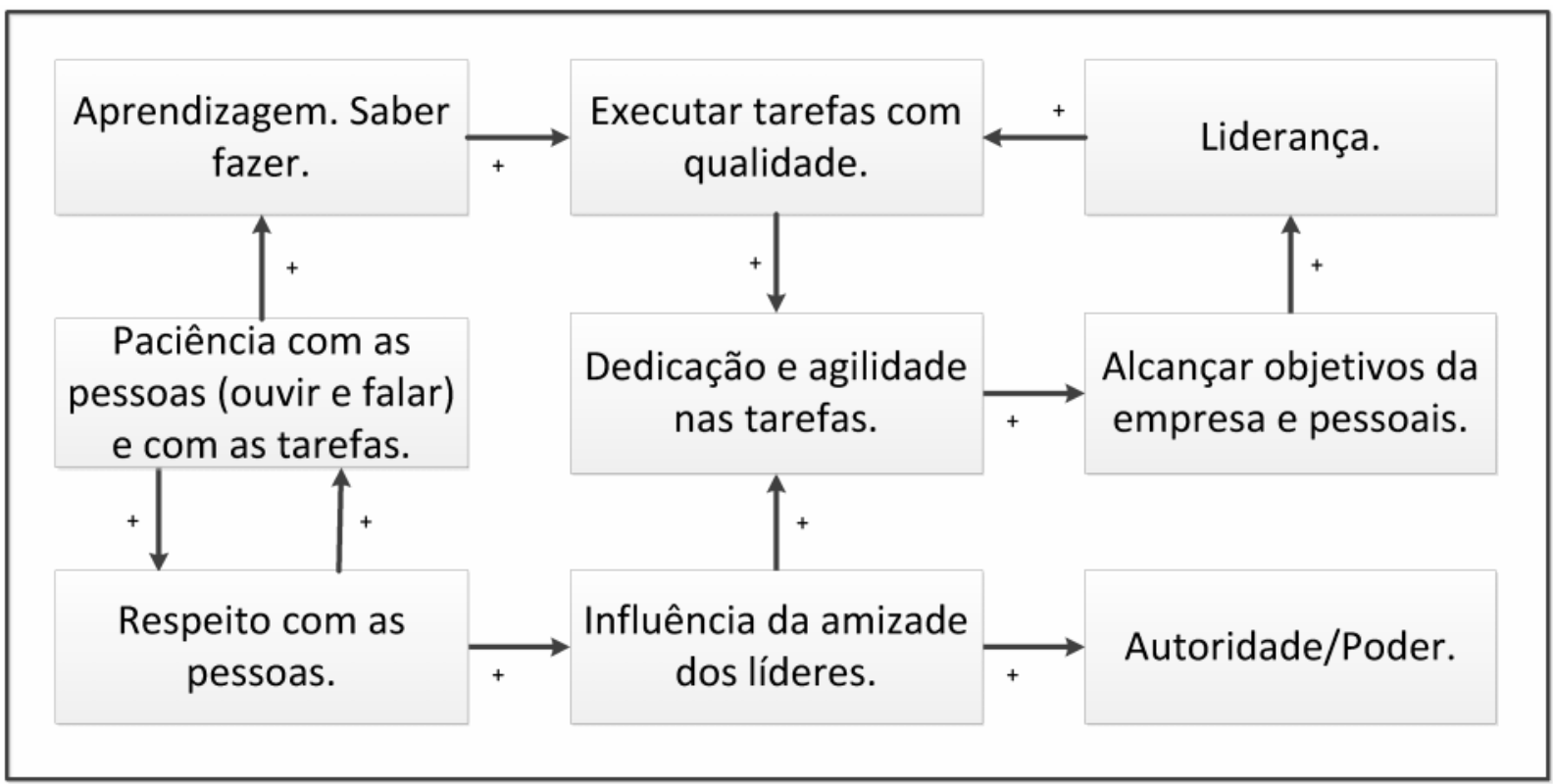

Fonte: Elaborada pelos autores a partir dos resultados da pesquisa.

Ao analisar a Figura 2, percebe-se que a conexão central do mapa se refere à Dedicação e agilidade nas tarefas, e que está relacionada a outras atitudes necessárias. Nota-se que tais atitudes são as características essenciais para o exercício da liderança na empresa Q-Frango. Estas características são percebidas como decorrentes de Executar tarefas com qualidade e da Influência da amizade dos líderes. Estas duas, por sua vez, são decorrentes da Paciência com as pessoas (ouvir e falar) e com as tarefas, e retroalimentada pela Liderança.

A Paciência com as pessoas (ouvir e falar) e com as tarefas, por sua vez, emerge como outra característica essencial, pois influencia em todas as outras atitudes identificadas no mapa. Destaca-se a retroalimentação com o Respeito com as pessoas e a influência na Aprendizagem. Saber fazer, e esta influenciando em Executar tarefas com qualidade, e aquela em Influência da amizade dos líderes. Além disso, pode-se inferir que a Liderança é percebida como decorrente de Alcançar objetivos da empresa e pessoais, e esta é influenciada pela questão central: Dedicação e Agilidade nas tarefas.

Chamou a atenção sobremaneira dos pesquisadores, que os participantes elegeram a característica Autoridade/Poder como necessária, mas esta não impactou diretamente na Liderança. Tal característica, que na percepção dos participantes é Revista Eletrônica de Estratégia \& Negócios, Florianópolis, v.7, n.2, mai./ago. 2014. 
decorrente da "influência da amizade dos líderes", demonstra que mesmo a autoridade formal é altamente influenciada por questões atinentes às relações humanas, sinalizando que líderes com a autoridade e o poder formalizados, provavelmente terão maiores facilidades no domínio da liderança se obtiverem, também, a influência da amizade de outros colegas.

\section{FEEDBACK DOS PARTICIPANTES}

Na terceira fase do estudo, que sucedeu a criação dos mapas coletivos, tanto pelas representações sociais (Figura 1) quanto pelo Self Q (Figura 2), as duas representações foram expostas a todos os entrevistados e, então, foi proposta uma discussão. Os dois mapas foram devolvidos aos entrevistados, separados por grupo de participação, e foram convidados a avaliar, em uma escala do tipo Likert de 1 a 5, variando de 1 . Totalmente Insatisfeito a 5. Totalmente Satisfeito em relação à medida que cada mapa conseguiu capturar (ou representar) as características indicadas pelos participantes no início dos trabalhos. Os participantes dos grupos um e dois afirmaram estar bastante satisfeitos com a capacidade representativa dos gráficos, e que estes conseguiram representar o que haviam dito.

Os participantes do grupo que operacionalizou as representações sociais atribuíram uma pontuação média de cinco (desvio padrão igual a 0) para o seu grau de satisfação (totalmente satisfeitos) com os resultados do mapa. O mapa causal construído a partir das nove conexões enigmáticas (Self Q), igualmente ao anterior, resultou em um nível de satisfação médio igual a cinco (desvio padrão igual a 0). Nesta fase do trabalho, oportunizou-se, pela primeira vez, que os membros do primeiro e do segundo grupo pudessem examinar os resultados uns dos outros, comparativamente.

Do mesmo modo, as duas representações foram apresentadas para o terceiro grupo pela primeira vez. Após as explanações sobre as metodologias e demonstrações dos resultados encontrados, todos os participantes foram convidados a preencher um pequeno questionário projetado para identificar se existiam percepções de grandes diferenças entre as duas representações. A totalidade dos participantes do terceiro grupo afirmou que não 
percebia grandes diferenças entre as duas representações. Ou seja, o mesmo resultado foi encontrado em relação aos outros dois grupos.

\section{PRINCIPAIS RESULTADOS}

O objetivo inicial do presente estudo foi verificar diferenças e similaridades entre dois métodos cognitivos de análise organizacional (pela Teoria das Representações Sociais (TRS) e pelo Self Q), e também para descrever fatores relevantes do pensamento cognitivo nas organizações, observando um contexto organizacional específico, neste caso, a empresa denominada Q-Frango. Neste sentido, procurou-se obter uma oportunidade que permitisse as duas abordagens teóricas e metodológicas, que os pesquisadores divergiram e observaram similaridades: uma que está enraizada na tradição da psicologia social e na sociologia francesa e que é baseada na análise de dados textuais reunidos por visões gerais e abertas (Teoria das Representações Sociais - TRS), e outra derivada de uma combinação da psicologia e da cibernética que é baseada em um ciclo de autoentrevistas (Self Q) (NICOLINI, 1999).

As duas representações permitiram que os membros dos grupos fizessem uma avaliação de seu próprio ser dentro da organização, bem como o impacto das suas ações nos colegas. Durante a discussão entre todos os participantes a respeito dos resultados encontrados, quando percebidos os fatores de retroalimentação como, por exemplo, o Respeito com as pessoas e a Paciência com as pessoas (ouvir e falar) e com as tarefas, os benefícios para ambos os sujeitos foram associados. Esta retroalimentação de temas foi percebida também como um processo de comunicação e de interações entre os colegas no chão de fábrica.

Em relação às capacidades das duas metodologias captarem representações semelhantes, acredita-se que os resultaram ratificaram os achados de Nicolini (1999). Devese, no entanto, resguardar algumas propriedades de cada método. Porém, de maneira geral, entende-se que os dois métodos cumpriram com as competências a que se propunham.

Vejamos, a paciência com as pessoas (ouvir e falar) e com as tarefas, que surgiu como elemento essencial, conforme demonstrado na Figura 2, pode ser facilmente 
associada com o Ouvir com atenção, que emergiu como um dos elementos centrais no método das representações sociais (Figura 1). Da mesma forma, o Respeito com as pessoas (Figura 2), retroalimentado por Paciência com as pessoas (ouvir e falar) e com as tarefas, assemelha-se com Ser bem-educado com as pessoas, também elemento central do método 1 (Figura 1). Por último, Ser responsável com as tarefas da empresa (Figura 1) está associado com Dedicação e agilidade nas tarefas (Figura 2), que foi percebido como meio para alcançar objetivos da empresa e pessoais e, consequentemente, ter sucesso na Liderança.

Um aspecto que chama a atenção em ambas as percepções dos grupos é o compromisso com os objetivos e com as tarefas da empresa. Na percepção dos pesquisadores, existe um elevado compromisso por parte dos participantes com estas questões. Por outro lado, nota-se que a necessidade de haver e de desenvolver habilidades com as relações humanas se fez fortemente presente nos resultados do presente estudo.

\section{CONSIDERAÇÕES FINAIS}

Como proposto por Nicolini (1999), os mapas das representações sociais e do Self $Q$ são instrumentos extremamente úteis, porque descrevem a maneira como certas classes de fenômenos são interpretadas, tornando-se a base para a autorreflexão que produz o aprendizado e a mudança organizacionais. Assim sendo, este trabalho oportuniza a reflexão sobre duas técnicas para captação de tais fenômenos, contribuindo para a validação de suas metodologias, conforme preconizado no estudo seminal desenvolvido por Nicolini (1999).

A utilização destas metodologias em ambientes empresariais pode repercutir em benefícios para os gestores das organizações. Entendidas como representações, elas podem contribuir na identificação do pensamento dos colaboradores, servindo, por exemplo, de base para a construção de estratégias e de programas de recursos humanos, voltados à consolidação de uma cultura organizacional saudável, à melhoria do clima organizacional e ao aumento na performance dos colaboradores.

De maneira a contribuir com o exposto anteriormente, exemplifica-se que os resultados do trabalho foram apresentados também à Diretoria de Recursos Humanos da 
empresa Q-frango. Esta, após analisar os mesmos, concordou com as duas representações. Além de acreditarem que a essência do trabalho, no que se refere às questões das relações humanas, é decorrente da cultura e de práticas da alta administração da empresa, substituídas há poucos meses, e vislumbram a necessidade de estabelecer uma agenda futura para potencializar o desempenho dos colaboradores e, por decorrência disso, da própria empresa. Inclusive, o Diretor de Recursos Humanos da referida empresa entende que este trabalho não só é validado sob o ponto de vista gerencial, bem como deverá ser ampliado ao nível estratégico, e os seus resultados servirão como base para o desenvolvimento de habilidades e competências que deverão ser evoluídas em futuras ações de capacitação e aperfeiçoamento.

Por outro lado, concorda-se com Narayan e Fahey (1990), que sugerem que a correlação entre a percepção do verdadeiro não é perfeito em mapas causais, mas é moldada pela natureza do discurso público e do contexto no qual ele ocorre. Projetar os resultados encontrados como algo conclusivo é uma temeridade, pois, dependendo do contexto, e alguns reflexos disso podem ter ocorrido neste estudo, como, por exemplo, o mesmo ter acontecido próximo a algumas demissões, incidência de acidentes de trabalho, folha de pagamento e discussões entre líderes e funcionários, pode ter interferido nas percepções e opiniões dos participantes. Mesmo assim, os resultados mostraram-se coerentes. Por isso, futuros trabalhos focando essas metodologias precisam ser projetados e operacionalizados, de maneira a colaborar, tanto com a academia quanto com o meio gerencial.

\section{REFERÊNCIAS}

ABRIC, J. C. A theoretical and experimental approach to the study of social representations in a situation of interaction. In: FARR, R. M.; MOSCOVICl, S. (eds.). Social representations. Cambridge: Cambridge University Press, p. 169-183, 1984.

ABRIC, J. C. Central system, peripheral system: their functions and roles in the dynamics of social representations. Papers on Social Representations, v. 2, n. 2, p. 75-78, 1993.

ABRIC, J. C. Les représentations sociales: aspects théorique. In: ABRIC, J. C. (ed.). Pratiques sociales et représentations. Paris: Presses Universitaire de France, p. 11-35, 1994. 
UM ESTUDO SOBRE TÉCNICAS DE MAPEAMENTO DA COGNIÇÃO ORGANIZACIONAL Lasier Gorziza de Souza - Heitor José Cademartori Mendina - Gabriel Sperandio Milan - Claudia Cristina Bitencourt

ABRIC, J. C. Specific processes of social representations. Papers on Social Representations, v. 5, n. 1, p. 77-80, 1996.

AXELROD, R. M. The structure of decision: cognitive maps of political elites. Princeton: University of Princeton Press, 1976.

BARNARD, C. I. The functions of the executive. Cambridge: Harvard University Press, 1938. BARDIN, L. Análise de conteúdo. Lisboa: Edições 70, 2004.

BOUGON, M. Uncovering cognitive maps: the Self Q technique. In: MORGAN, G. (ed.). Beyond method: strategies far social research. Beverly Hills: Sage Publications, p. 112-144, 1983.

BOUGON, M. Uncovering cognitive maps. Privately printed handbook. $5^{\text {th }}$ edition. Pennsylvania State University, 1986.

BOUGON, M. Congregate cognitive maps: a unified dynamic theory of organization strategy. Journal of Management Studies, v. 29, n. 3, p. 369-387, 1992.

BOUGON, M.; BAIRD, N.; KOMOCAR, J. M.; ROSS, W. Identifying strategic loops: the Self Q interview. In: HUFF, A. (ed.). Mapping strategic thought. Chichester: Wiley, p. 327-354, 1990.

BOUGON, M.; WEICK, K. E.; BINKHORST, D. Cognition in organizations: an analysis of the Utrecht Jazz Orchestra. Administrative Science Quarterly, v. 22, p. 606-639, 1977.

CYERT, R.; MARCH, J. A behavioral theory of the firm. Englewood Cliffs: Prentice-Hall, 1963.

DEGENNE, A. ; VERGÈS, P. Introduction à "analyse de similitude". Revue Française de Sociologie, v. 16, p. 471-512, 1973.

DI GIACOMO, J. P. Rappresentazioni sociali e movimenti collettivi. Naples: Liguori, 1985.

EDEN, C.; SPENDER, J. C. Managerial and organizational cognition: theory, methods and research. London: Sage Publications, 1998.

FARR, R. M. Social representations: a French tradition of research. Journal for the Theory of Social Behavior, v. 17, n. 4, p. 343-369, 1987.

FARR, R. M. Theory and method in the study of social representations. In: BREAKWELL, G. M.; CANTER, D. (eds.). Empirical approaches to social representations. Oxford: Clarendon Press, p. 15-38, 1993.

FARR, R. M.; MOSCOVICI, S. Social representations. Cambridge: Cambridge University Press, 1984.

FIOL, M.; HUFF, A. S. Maps for managers: where are we where do we go from here? Journal of Management Studies, v. 29, n. 3, p. 267-286, 1992.

Revista Eletrônica de Estratégia \& Negócios, Florianópolis, v.7, n.2, mai./ago. 2014. 
FLAMENT, C. L. Analyse de similitude: une technique pour le recherches sur les représentations sociales. Cahier de Psyxitologie Cognitive, v. 18, n. 1, p. 423-429, 1981.

FLAMENT, C. L. Pratique et représentations sociales. In: BEAUVOIS, J. L.; JOULE, R. V.; MONTEIL, J. M. (eds.). Perspectives cognitives et conduites sociales. Cousset: DelVal, 143-150, 1987.

FLAMENT, C. L. Consensus, salience and necessity in social representations: technical note. Papers on Social Representations, v. 3, n. 2, p. 97-105, 1994a.

FLAMENT, C. L. Structure, dynamic et transformation sans rupture d'une représentation sociale. In: ABRIC, J. C. (ed.). Pratiques sociales et représentations. Paris: Presses Universitaire de France, 1994b.

GHERARDI, S. Competence - the symbolic passe-partout for change - in a learning organization. Scandinavian Journal of Management, v. 4, p. 373-393, 1998.

HENNINK, M.; HUTTER, I.; BAILEY, A. Qualitative research methods. Thousand Oaks: Sage Publications, 2011.

HUFF, A. S. Mapping strategic thought. Chichester: Wiley, 1990.

JODELET, D. Représentations sociales: un domaine en expansion. In: JODELET, D. (ed.). Les représentations sociales. Paris: Presses Universitaires de France, p. 31-61, 1989.

JODELET, D. Madness and social representations. Hemel Hampstead: Harvester Wheatsheaf, 1991.

JODELET, D. Representações sociais: um domínio em expansão. In: JODELET, D. (org.). As representações sociais. Rio de Janeiro: EDUERJ, p. 17-44, 2001.

KING, N.; HORROCKS, C. Interviews in qualitative research. Thousand Oaks: Sage Publications, 2010.

MARCH, J.; SIMON, H. Organizations. New York: Wiley, 1958.

MORGAN, G. Images of organization. Newbury Park: Sage Publications, 1986.

MOSCOVICI, S. Preface in santé et maladie: analyse d'une représentation sociale. Paris: Mouton, 1969.

MOSCOVICI, S. On social representations. In: FORGAS, J. P. (ed.). Social cognitions: perspectives on everyday understanding. London: Academic Press, 1981.

MOSCOVICI, S. The phenomenon of social representations. In: FARR, R.; MOSCOVICI, S. (eds.). Social representations. Cambridge: Cambridge University Press, p. 3-69, 1984.

MOSCOVICl, S. Comment on Potter and Linton. British Journal of Social Psychology, v. 24, p. 91-93, 1985.

Revista Eletrônica de Estratégia \& Negócios, Florianópolis, v.7, n.2, mai./ago. 2014. 
MOSCOVICl, S. Answers and questions. Journal for the Theory of Social Behavior, v. 17, n. 4, p. 513-529, 1987.

MOSCOVICl, S. Notes towards a description of social representations. European Journal of Social Psychology, v. 18, n. 3, p. 211-250, 1988.

MOSCOVICl, S. Social representation: explorations in social psychology. Cambridge: Polity Press, 2000.

NARAYANAN, V. K.; FAHEY, L. Evolution of revealed causal maps during decline: a case study of admiral in mapping strategic thought. In: HUFF, A. (ed.). Mapping strategic thought. Chichester: Wiley, p. 109-134, 1990.

NICOLINI, D. Metodi cognitivi di analisi organizzativa: una rassegna critica. Psicologia del Lavoro, v. 98, p. 34-58, 1995.

NICOLINI, D. Comparing methods for mapping organizational cognition. Organization Studies, v. 20, n. 5, p. 833-860, 1999.

RIBEIRO, J. L. D.; MILAN, G. S. Planejando e conduzindo entrevistas individuais. In: RIBEIRO, J. L. D.; MILAN, G. S. (eds.). Entrevistas individuais: teoria e aplicações. Porto Alegre: FEEng/UFRGS, cap. 1, p. 9-22, 2004.

SÁ, C. P. Sobre o núcleo central das representações sociais. Petrópolis: Vozes, 1996. SÁ, C. P. A construção do objeto de pesquisa em representações sociais. Rio de Janeiro: EDUERJ, 1998.

SCHNEIDER, S.; ANGELMAR, R. Cognition in organizational analysis: who's minding the store? Organization Studies, v. 14, n. 3, p. 32-374, 1993.

SCHREIER, M. Qualitative content analysis in practice. London: Sage Publications, 2012.

SCOTT, G.; GARNER, R. Doing qualitative research: designs, methods, and techniques.

$1^{\text {st }}$ edition. Upper Saddle River: Pearson, 2013.

STRATI, A.; NICOLINI, D. Cognitivism in organization studies. In: ORTMANN, G.; SYDOW, J.; TURK, K. (eds.). Die rückkehr der gesellschaft: theorien der organisation. Opladen: Westdeutschen Verlag, p. 388-416, 1997.

VERGÈS, P. L'evocation de lárgent: une méthode pour la definition du noyau central dune representation. Bulletin de Psychologie, v. 45, n. 405, p. 203-209, 1992.

VERGÈS, P.; TYSZKA, T. Noyau central, saillance et propriétés structurales. Papers on Social Representations, v. 3, n. 1, p. 3-12, 1994.

WALSH, J. P. Managerial and organizational cognition: notes from a trip down memory lane. Organization Science, v. 6, n. 3, p. 280-334, 1995. 
UM ESTUDO SOBRE TÉCNICAS DE MAPEAMENTO DA COGNIÇÃO ORGANIZACIONAL Lasier Gorziza de Souza - Heitor José Cademartori Mendina - Gabriel Sperandio Milan - Claudia Cristina Bitencourt

WEICK, K. E.; BOUGON, M. Organizations as cognitive maps: charting ways of success and failure. In: SIMS Jr., H. P.; GIOIA, D. (eds.). The thinking organization. San Francisco: JosseyBass, p. 102-135,1986.

Revista Eletrônica de Estratégia \& Negócios, Florianópolis, v.7, n.2, mai./ago. 2014. 